\title{
ARTICLE Somatic and germline mutations in the tumor suppressor gene PARK2 impair PINK1/Parkin-mediated mitophagy in lung
} cancer cells

\author{
Zeng-li Zhang ${ }^{1}$, Na-na Wang ${ }^{2}$, Qi-lian Ma ${ }^{2}$, Yang Chen ${ }^{2}$, Li Yao ${ }^{2}$, Li Zhang ${ }^{5}$, Qiu-shi Li ${ }^{6}$, Min-hua Shi ${ }^{1}$, Hong-feng Wang ${ }^{2}$ and \\ Zheng Ying $2,3,4$
}

\begin{abstract}
PARK2, which encodes Parkin, is a disease-causing gene for both neurodegenerative disorders and cancer. Parkin can function as a neuroprotector that plays a crucial role in the regulation of mitophagy, and germline mutations in PARK2 are associated with Parkinson's disease (PD). Intriguingly, recent studies suggest that Parkin can also function as a tumor suppressor and that somatic and germline mutations in PARK2 are associated with various human cancers, including lung cancer. However, it is presently unknown how the tumor suppressor activity of Parkin is affected by these mutations and whether it is associated with mitophagy. Herein, we show that wild-type (WT) Parkin can rapidly translocate onto mitochondria following mitochondrial damage and that Parkin promotes mitophagic clearance of mitochondria in lung cancer cells. However, lung cancer-linked mutations inhibit the mitochondrial translocation and ubiquitin-associated activity of Parkin. Among all lung cancer-linked mutants that we tested, A46T Parkin failed to translocate onto mitochondria and could not recruit downstream mitophagic regulators, including optineurin (OPTN) and TFEB, whereas N254S and R275W Parkin displayed slower mitochondrial translocation than WT Parkin. Moreover, we found that deferiprone (DFP), an iron chelator that can induce mitophagy, greatly increased the death of A46T Parkin-expressing lung cancer cells. Taken together, our results reveal a novel mitophagic mechanism in lung cancer, suggesting that lung cancer-linked mutations in PARK2 are associated with impaired mitophagy and identifying DFP as a novel therapeutic agent for PARK2-linked lung cancer and possibly other types of cancers driven by mitophagic dysregulation.
\end{abstract}

Keywords: autophagy; mitophagy; Parkin; ubiquitin; cancer

Acta Pharmacologica Sinica (2020) 41:93-100; https://doi.org/10.1038/s41401-019-0260-6

\section{INTRODUCTION}

Parkin is an important E3 ubiquitin ligase that is activated by phosphorylated ubiquitin and promotes the polyubiquitination of various substrates [1]. Under normal conditions, Parkin localizes throughout the nucleus and cytosol, and it can translocate onto damaged mitochondria to promote the ubiquitination of mitochondrial proteins and trigger mitophagy under stress conditions [2]. Loss-of-function mutations in PARK2, the gene encoding Parkin, cause Parkinson's disease (PD) [3]. However, Parkin knockout mice did not display any neurodegenerative phenotype but developed spontaneous hepatocellular carcinoma and tumor growth $[4,5]$, indicating the complexity of Parkin's functions and associated disease mechanisms. Moreover, the expression level of Parkin was found to be decreased in several human cancers, including lung cancer $[6,7]$, and somatic mutations in PARK2 were found in glioblastoma and lung cancer $[8,9]$. Importantly, overexpression of Parkin inhibited cell growth and proliferation in multiple cancer cell lines [10-12], indicating that Parkin can act as a tumor suppressor and that its dysfunction may contribute to oncogenesis in somatic cells.

Autophagy is an important degradation pathway responsible for cell homeostasis [13]. Although the role of autophagy in cancer cell survival and death has been extensively explored, the relationship between cancer and the selective forms of autophagy, including mitophagy, is not yet well understood [14]. Parkin acts as a crucial mediator in mitophagy regulation [2, 14]. Recent research advances in mitophagy have revealed that under normal conditions, Parkin displays an autoinhibited state, since the active site of Parkin is sealed by the REP domain [15], whereas under disease conditions or mitochondrial stress, Parkin is phosphorylated by mitochondrial PINK1 at the N-terminal ubiquitin-like (UBL) domain and interacts with phosphorylated ubiquitin on the mitochondrial outer membrane [16]. After a series

\footnotetext{
${ }^{1}$ Department of Respiration, The Second Affiliated Hospital of Soochow University, Suzhou 215004, China; ${ }^{2}$ Jiangsu Key Laboratory of Neuropsychiatric Diseases and College of Pharmaceutical Sciences, Soochow University, Suzhou 215123, China; ${ }^{3}$ School of Pharmacy, Key Laboratory of Molecular Pharmacology and Drug Evaluation (Yantai University), Ministry of Education, Yantai University, Yantai 264005, China; ${ }^{4}$ Jiangsu Key Laboratory of Preventive and Translational Medicine for Geriatric Diseases, College of Pharmaceutical

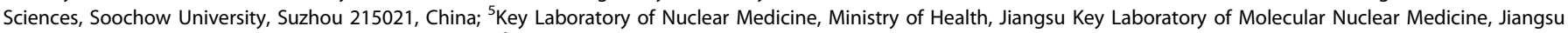
Institute of Nuclear Medicine, Wuxi 214063, China and ${ }^{6}$ National University of Singapore (Suzhou) Research Institute, Suzhou 215123, China

Correspondence: Hong-feng Wang (wanghongfeng@suda.edu.cn) or Zheng Ying (zheng.ying@suda.edu.cn)

These authors contributed equally: Zeng-li Zhang, Na-na Wang
}

Received: 22 March 2019 Accepted: 21 May 2019

Published online: 8 July 2019 
of conformational changes, the RING finger E3 catalytic center is exposed, and Parkin acts as an activated E3 ubiquitin ligase $[2,15]$. According to recent studies, Parkin can facilitate the ubiquitination of outer mitochondrial membrane (OMM) proteins and therefore enhance the recognition of polyubiquitinated mitochondria by mitophagic receptors, such as optineurin (OPTN) or NDP52 $[17,18]$. These receptors can bind to autophagosomal proteins, such as LC3 and target ubiquitinated mitochondria for lysosomal degradation [19]. Moreover, a recent report showed that TFEB, the master transcription factor regulating the expression of autophagic and lysosomal genes, is a downstream regulator of mitophagy [20]. The above process, which is very powerful for clearing damaged mitochondrial and is the most well-understood form of mitophagy, is called PINK1/Parkinmediated mitophagy [2].

According to previous studies, somatic PARK2 mutations in lung cancer include the A46T, N254S and H279P mutations [8], while germline mutations in PARK2 are found in familial lung cancer [9]. It is worth noting that all these lung cancer-linked mutations are highly enriched in ubiquitin-associated domains of Parkin. In detail, the A46T mutation occurs in the UBL domain, whereas the N254S, R275W and H279P mutations occur in the RING1 domain of Parkin [8, 9]. These findings suggest that Parkinassociated ubiquitin regulation may contribute to the disease pathogenesis underlying lung cancer. However, whether these mutations affect Parkin-mediated mitophagy and the potential role of this alteration in lung cancer tumorigenesis remain unknown. In this study, we showed that these lung cancerlinked mutations abolish the recognition of damaged mitochondria by autophagosomes due to the loss of mitochondrial ubiquitination driven by Parkin. Importantly, we identified that deferiprone (DFP), which is known as a mitophagy enhancer [21], can induce cell death in lung cancer cells expressing mutant Parkin.

\section{MATERIALS AND METHODS}

Plasmid construction and siRNAs

The FLAG, FLAG-Parkin, HA-Ub, GFP-OPTN, mCherry-C1 and GFPTFEB plasmids were described before [22-27]. mCherry-Parkin was generously provided by Richard Youle (Addgene \#23956); BFPmito was generously provided by Gia Voeltz (Addgene \#49151). FLAG-tagged and mCherry-tagged A46T, N254S and R275W Parkin mutants were generated by site-directed mutagenesis with a MutanBEST kit (Takara, Shiga, Japan). pcDNA3.1-mt-Keima was generated by inserting mKeima cDNA into the vector at the Kpnl and EcoRI sites. All constructs were verified by sequencing. Small interfering RNAs targeting human OPTN and PINK1 were synthesized by Shanghai GenePharma.

Cell culture and treatments

Human alveolar epithelial cells (A549) were cultured in DMEM (Gibco, Grand Island, NY, USA) containing 10\% FBS (Gibco), $100 \mathrm{U} / \mathrm{mL}$ penicillin and $100 \mathrm{mg} / \mathrm{mL}$ streptomycin (Gibco). Cells were transfected with DNA plasmids using Lipofectamine 2000 (Invitrogen, Carlsbad, CA, USA) $24 \mathrm{~h}$ after splitting and with siRNA using Lipofectamine RNAiMAX (Invitrogen) upon splitting. Propidium iodide (PI, Sigma, St. Louis, MO, USA; $0.5 \mathrm{mg} / \mathrm{mL}$ ) staining was used to detect dead cells. Antimycin A1, chloroquine (CQ), CCCP and Hoechst were purchased from Sigma. Oligomycin was purchased from Calbiochem. Bafilomycin A1 (Bafi A1) was obtained from Selleckchem, and MitoTracker Red CMXRos was purchased from Thermo Fisher Scientific (Waltham, MA, USA).

Immunoblot analysis and immunofluorescence

For immunoblot analysis, cells were lysed with cell lysis buffer $[22,25,27]$. Proteins were then separated by SDS-PAGE and transferred onto PVDF membranes (Millipore, Billerica, MA, USA).
The primary antibodies anti-GAPDH and anti-Hsp60 were purchased from Proteintech. Horseradish peroxidase-conjugated sheep anti-mouse antibody (Jackson ImmunoResearch Laboratories, PA, USA) was used as the secondary antibody. Proteins were detected using an ECL detection kit (Thermo Fisher Scientific). Immunoblot analysis was performed with a standard protocol as previously reported [28-33]. For immunofluorescence, cells were fixed with $4 \%$ paraformaldehyde, permeabilized with $0.1 \%$ Triton X-100 and blocked with $0.1 \%$ FBS. Then, cells were incubated sequentially with primary antibodies and secondary antibodies. Anti-HA (Santa Cruz, CA, USA), anti-FLAG (Sigma) and anti-TOM20 (Proteintech, Rosemont, IL, USA) antibodies were used as primary antibodies. Alexa Fluor 405-conjugated goat antirabbit $\lg G(\mathrm{H}+\mathrm{L})$ (Invitrogen) and Alexa Fluor 594-conjugated goat anti-mouse lgG (Proteintech) were used as secondary antibodies. Eventually, cells were visualized using a Nikon [34] or Zeiss LSM710 confocal microscope [35, 36]. Briefly, the imaging analysis was performed by researchers who were blinded to the study, and fluorescence was quantified using ImageJ software. For live cell imaging, cells were stained with MitoTracker Red CMXRos and Hoechst as described in Fig. S4.

Statistical analysis

Parkin and OPTN mitochondrial localization was determined by visual inspection in $10 \mathrm{~min}$ intervals. PI-positive cells were considered nonviable. Data are presented as the means \pm SDs. Comparisons were performed using Prism 6.0 (GraphPad Software). $P<0.05$ was considered statistically significant.

\section{RESULTS}

Both somatic and germline mutations of Parkin in lung cancer impair Parkin-mediated mitophagy

Parkin, the E3 ubiquitin ligase, has been identified as a tumor suppressor in several cancers, including lung cancer. We were interested in several PARK2 lung cancer mutations, including the somatic mutations A46T and N254S, and a recurrent germline mutation, R275W (Fig. 1a) [8, 9]. To elucidate the effect of these Parkin mutations on mitophagy, we investigated the mitochondrial translocation of Parkin and mitochondrial clearance in mutant Parkin-overexpressing lung cancer (A549) cells. As shown in Fig. 1, wild-type (WT) Parkin translocated onto damaged mitochondria, followed by mitochondrial recruitment of the mitophagy receptor OPTN following A/O treatment, a standard method to induce cellular mitophagy [17]. However, Parkin with the lung cancer-related mutations-A46T, N254S and R275W - showed delayed mitochondrial translocation to different degrees (Fig. 1b, d). Long-term mitophagy induction resulted in complete clearance of mitochondria in cancer cells with WT Parkin. In contrast, mutant Parkin-expressing cells showed impaired mitochondrial clearance (Fig. 1c, e).

PINK1/Parkin-mediated mitophagy is blocked by Parkin mutations in lung cancer cells

Although PINK1/Parkin-mediated mitophagy has been extensively investigated, the relationship between mitophagy and lung tumorigenesis remains elusive. To understand the role of PINK1/ Parkin-mediated mitophagy in lung cancer cells, we used a longterm mitophagic degradation assay and investigated the turnover of mitochondrial proteins. Immunoblot analysis showed that the level of the mitochondrial matrix protein Hsp60 was decreased dramatically in cells expressing WT Parkin (Fig. 2a). Depletion of the mitophagic receptor OPTN caused a reduction in mitochondrial degradation, even in the presence of WT Parkin, which suggested that OPTN is a downstream mitophagic regulator of Parkin in lung cancer cells. Consistent with the fluorescence results shown in Fig. 1, A46T and N254S mutant Parkin had no significant effect on mitochondrial degradation, regardless of whether OPTN 
a

Parkin 1 Ubl

b
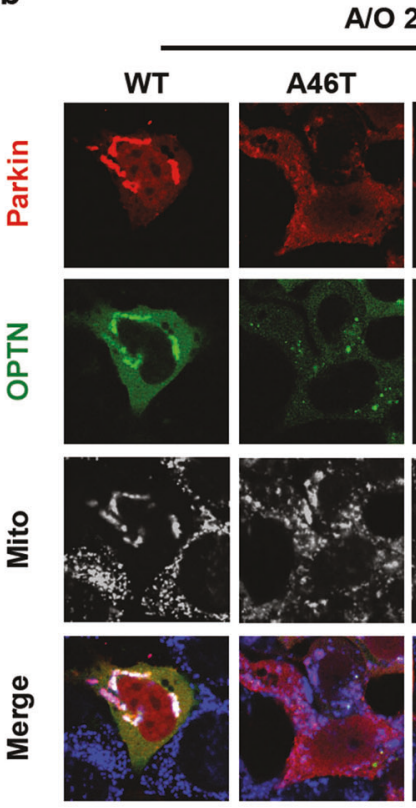

A/O 2 h
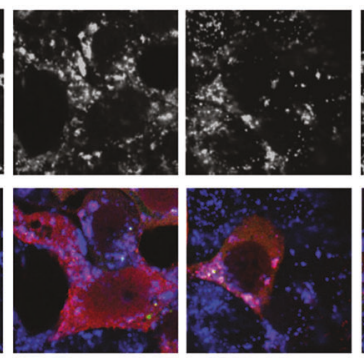

d

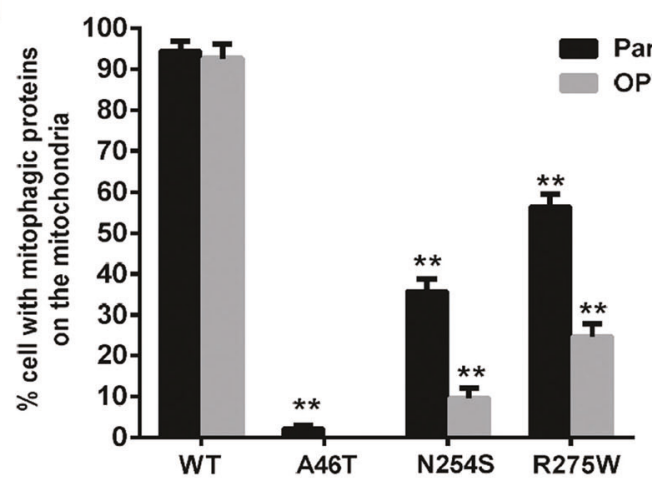

C
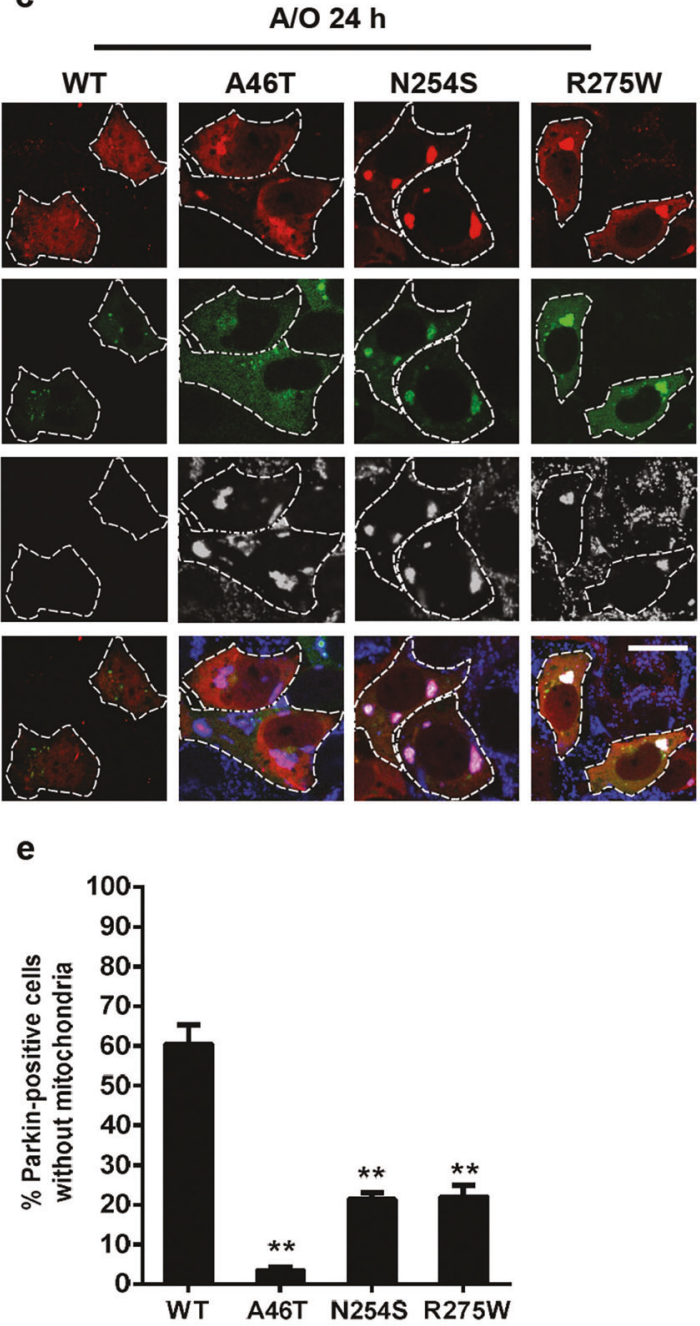

Fig. 1 Lung cancer-related mutations in Parkin lead to defects in mitochondrial translocation of Parkin and mitochondrial clearance in lung cancer cells. a Schematic diagram of Parkin and the pathogenic mutations in this study. $\mathbf{b}$ and $\mathbf{c}$ A549 cells were transfected with mCherrytagged wild-type (WT) and lung cancer-related mutant Parkin, along with EGFP-tagged OPTN. Twenty-four hours later, cells were treated with either $5 \mu \mathrm{M}$ antimycin $\mathrm{A}$ and $5 \mu \mathrm{M}$ oligomycin (A/O) for $2 \mathrm{~h}$ (b) or $1 \mu \mathrm{M} \mathrm{A} / \mathrm{O}$ for $24 \mathrm{~h}$ (c). Then, cells were subjected to immunocytochemistry using an anti-TOM20 antibody (blue) to detect mitochondria. Scale bar: $20 \mu \mathrm{m}$. d Quantification of Parkin and OPTN recruitment on mitochondria from three independent experiments as described in $\mathbf{b}$ is presented as the mean \pm SD values. Comparisons were made to the corresponding wild-type group (Parkin or OPTN); ${ }^{* *} P<0.01 ; t$-test. e Quantification of Parkin-positive cells without mitochondria from three independent experiments as described in $\mathbf{c}$ are presented as the mean \pm SD values. Comparisons were made to the wild-type group; ${ }^{* *} P<0.01$, $t$-test

was knocked down (Figs. 2a and S1a). To verify the effect of PINK1 on Parkin-associated mitophagy in lung cancer cells, we knocked down the crucial mitochondrial initiator PINK1, which is reported to phosphorylate Parkin and ubiquitin on mitochondria [2]. Compared to cells with endogenous PINK1 expression, PINK1deficient lung cancer cells did not exhibit mitochondrial clearance upon $\mathrm{A} / \mathrm{O}$ or CCCP treatment (Figs. $2 \mathrm{~b}$ and $\mathrm{S} 1 \mathrm{~b}$ ). To further test whether the degradation of the mitochondrial protein Hsp60 occurs through autophagy but not other degradation systems, we blocked the autophagy pathway with Bafi A1 or CQ. The results showed that these autophagy inhibitors abolished Parkinmediated mitophagy (Fig. 2c).
Lung cancer-linked mutations abolish mitochondrial recruitment of Parkin and ubiquitin and diminish nuclear translocation of TFEB during mitophagy

To further gain mechanistic insights into the failure of mitophagy driven by these Parkin mutations, we next performed a timecourse experiment to detect the speed of translocation of Parkin and OPTN onto mitochondria during mitophagy. As shown in Fig. 3, WT Parkin rapidly translocated to mitochondria in $\sim 2 \mathrm{~h}$, while N254S Parkin finished this process at $3 \mathrm{~h}$. Strikingly, A46T Parkin did not show strong mitochondrial localization within similar times (Fig. 3a, b). Therefore, we conclude that lung cancerlinked UBL mutations in Parkin lead to a significant failure of 


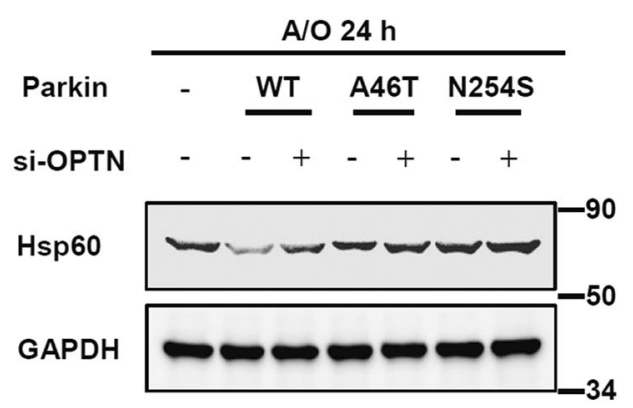

b

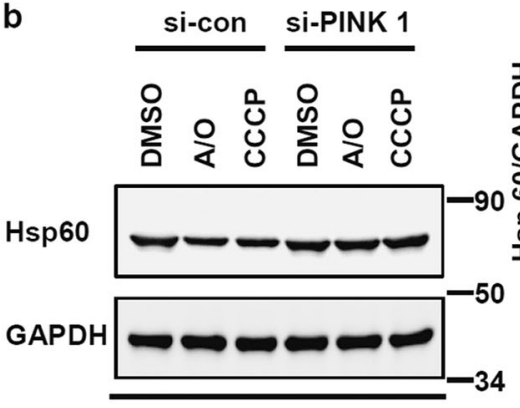

Parkin

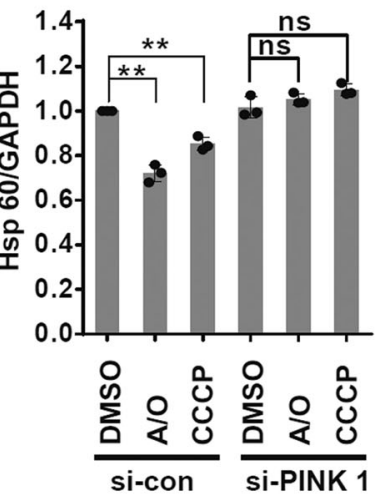

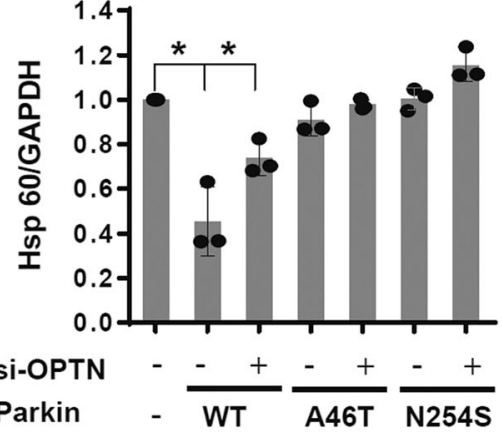

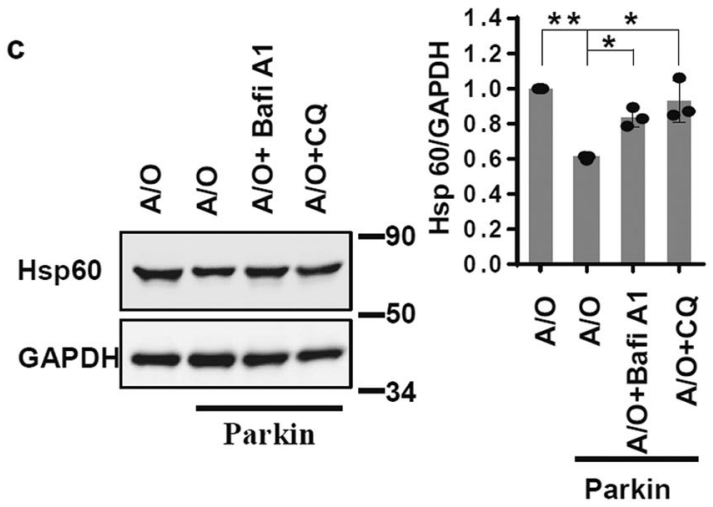

Fig. 2 Lung cancer-linked mutant Parkin fails to promote PINK1/Parkin-mediated mitophagic clearance of damaged mitochondria. a A549 cells were first transfected with control siRNA or siRNA targeting OPTN. Forty-eight hours later, cells were retransfected with wild-type, A46T or N254S Parkin. After $24 \mathrm{~h}$, cell lysates were treated with $1 \mu \mathrm{M}$ A/O for another $24 \mathrm{~h}$. b A549 cells were transfected with siRNA-targeting PINK1. Forty-eight hours later, cells were transfected with FLAG-tagged wild-type Parkin. After an additional $24 \mathrm{~h}$, cells were treated with $1 \mu \mathrm{M}$ A/O or $20 \mu \mathrm{M}$ CCCP for $24 \mathrm{~h}$. Cell lysates were used in immunoblot analysis. c A549 cells were transfected with FLAG-tagged Parkin. Twenty-four hours later, cells were incubated with $1 \mu \mathrm{M} \mathrm{A} / \mathrm{O}$ alone or in combination with $100 \mathrm{nM}$ bafilomycin $\mathrm{A} 1$ or $50 \mu \mathrm{M}$ chloroquine (CQ) for $24 \mathrm{~h}$. Quantitative data in a-c obtained from three independent experiments are shown as the means $\pm \mathrm{SDs} ;{ }^{*} P<0.05 ;{ }^{* \star} P<0.01$; ns, no significance; $t$-test

mitochondrial Parkin and receptor recruitment, whereas RING 1 mutations delay recruitment.

We next examined the translocation of TFEB, which reflects TFEB activity, during Parkin-mediated mitophagy, since it has been shown that TFEB functions downstream of Parkin during mitophagy [20]. We found that translocation of Parkin onto mitochondria was accompanied by TFEB nuclear translocation (Fig. 4a). However, the lung cancer-related Parkin mutations showed different effects. TFEB was expressed completely in the cytoplasm in cells with A46T Parkin expression and partially in the nucleus in cells with N254S Parkin expression (Fig. 4a). As an E3 ubiquitin ligase, Parkin is known to ubiquitinate damaged mitochondria and promote their clearance via autophagy. To determine the ubiquitin ligase activity of mutant Parkin, we tested the ubiquitination of mitochondria. The lung cancer-related Parkin mutants failed to ubiquitinate damaged mitochondria as WT Parkin did (Figs. 4b and S2). Our observation indicates that mutation of Parkin impaired its E3 ligase function in mitochondria.

Lung cancer mutant Parkin attenuates mitophagic flux

Based on the observation that mutated Parkin exhibits greatly delayed mitochondrial translocation and defective clearance of Hsp60, we next visualized mitophagic flux using a fluorescencesensitive probe, mt-Keima. According to previous studies, mtKeima is a mitochondria-located fluorescent protein that is $\mathrm{pH}$ stable. Keima emits different signals with changes in $\mathrm{pH}$. The signal peaks at $586 \mathrm{~nm}$ in acidic conditions, such as in the lysosomal lumen, or at $440 \mathrm{~nm}$ in neutral $\mathrm{pH}$ conditions. Therefore, acidic autolysosomes containing mitochondria, which reflects mitophagy, can be detected as " $561 \mathrm{~nm}$ " signals. Meanwhile, nonlysosomal mitochondria, which reflect the pool of mitochondria that are not targeted by mitophagy, can be detected as " 458 $\mathrm{nm}^{\prime \prime}$ signals $[37,38]$. We found that $561 \mathrm{~nm}$ signals existed when mitochondria were damaged for a short time (Fig. 5, left region, and Fig. S3), indicating that a portion of mitochondria were delivered to acidic lysosomes. Twenty-four hours of mitochondrial damage generated an overwhelming ratio of 561/458 $\mathrm{nm}$ signals in Parkin-expressing cells, indicating that most mitochondria were targeted by mitophagy (Fig. 5b, right region). Regarding the lung cancer-related Parkin mutations, the R275W and N254S mutations produced partial effects compared with those of WT Parkin, while the A46T mutation blocked most of the mitophagic flux.

Treatment with DFP prevents the survival of mutant Parkinexpressing lung cancer cells

According to previous reports, strong induction of mitophagy can trigger cancer cell death [39]. We found that long-term Parkinmediated mitophagy significantly induced cell death in lung cancer cells (Fig. 6a). However, expression of lung cancer-related Parkin mutants, especially the A46T mutant, resulted in decreased cell death compared to that seen with WT Parkin (Fig. 6a). Interestingly and importantly, we found that DFP, an iron chelator and mitophagy inducer available for clinical use [21], significantly increased the death of A46T Parkin-expressing cancer cells (Fig. 6b). Moreover, we found that in contrast to A/O treatment, DFP treatment did not affect mitochondrial dynamics and membrane potential (Fig. S4), indicating that DFP was not toxic to mitochondrial function and that it can induce cancer cell death 
a

A/O
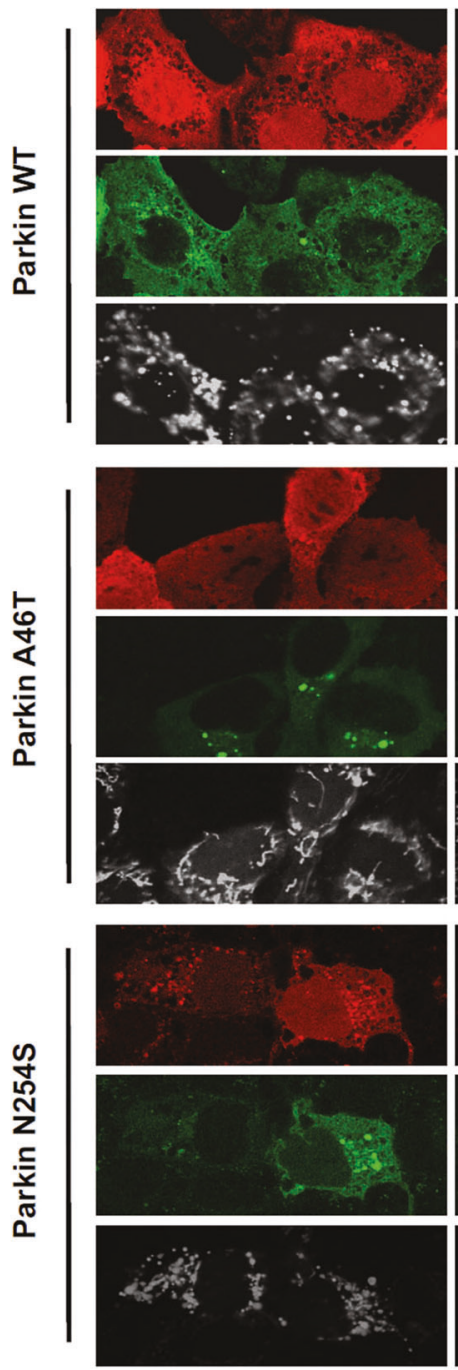

b
$1 \mathrm{~h}$
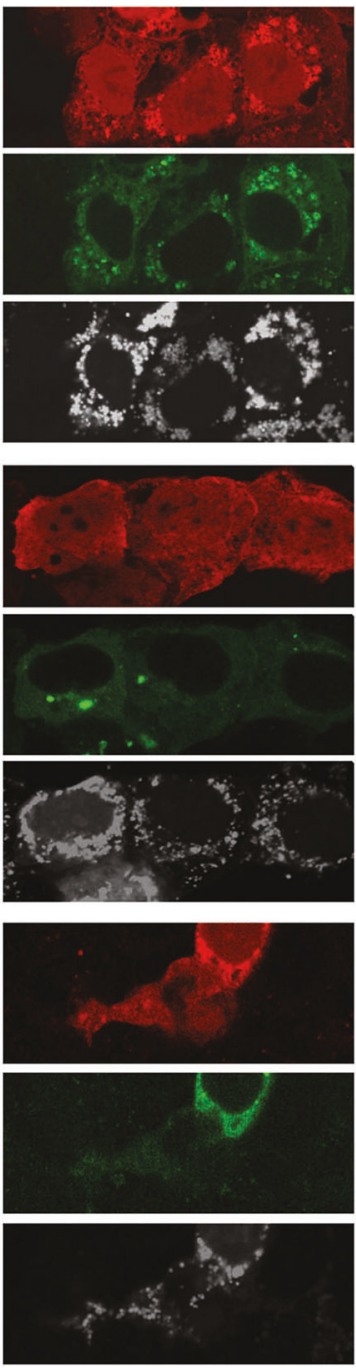

$2 \mathrm{~h}$
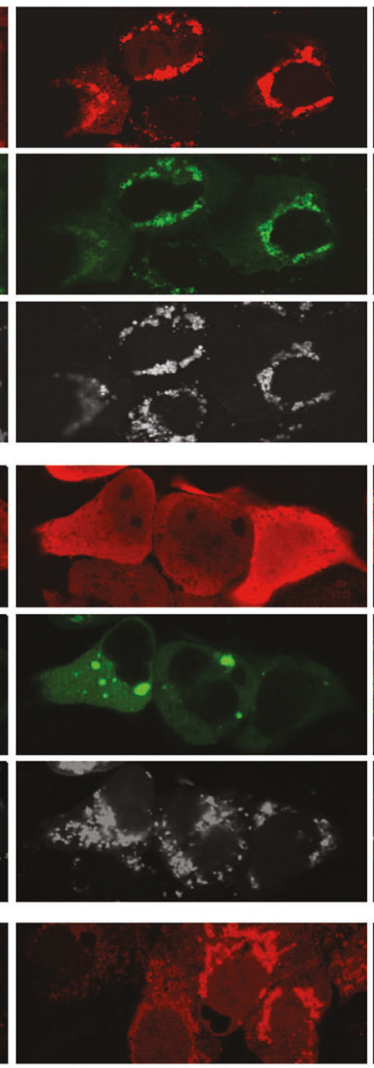
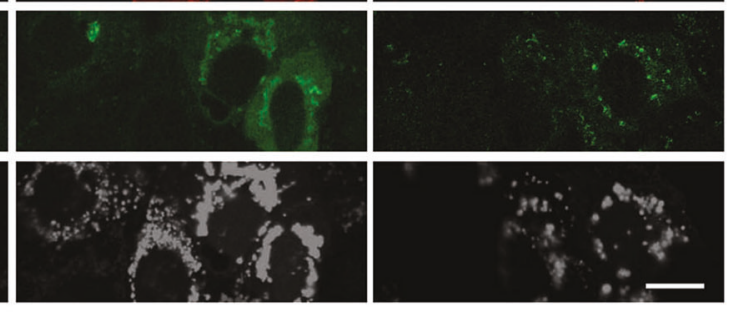

$3 \mathrm{~h}$
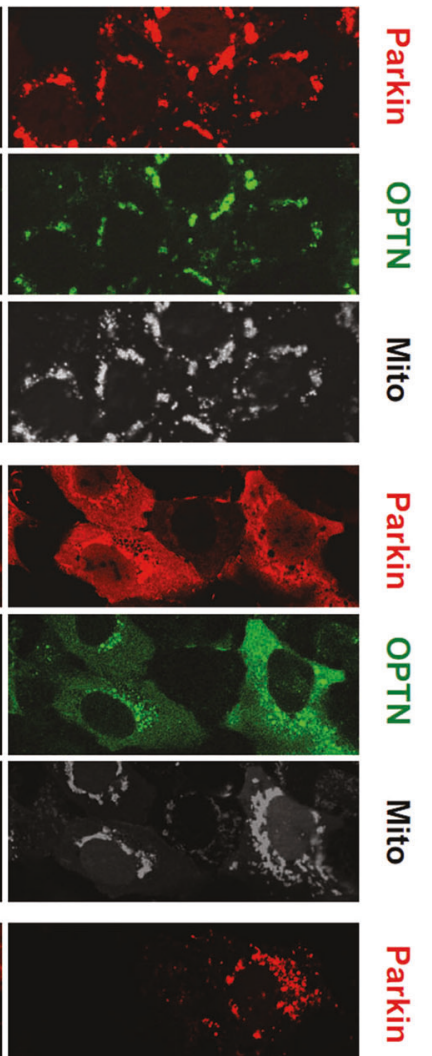

$\frac{0}{\frac{0}{2}}$

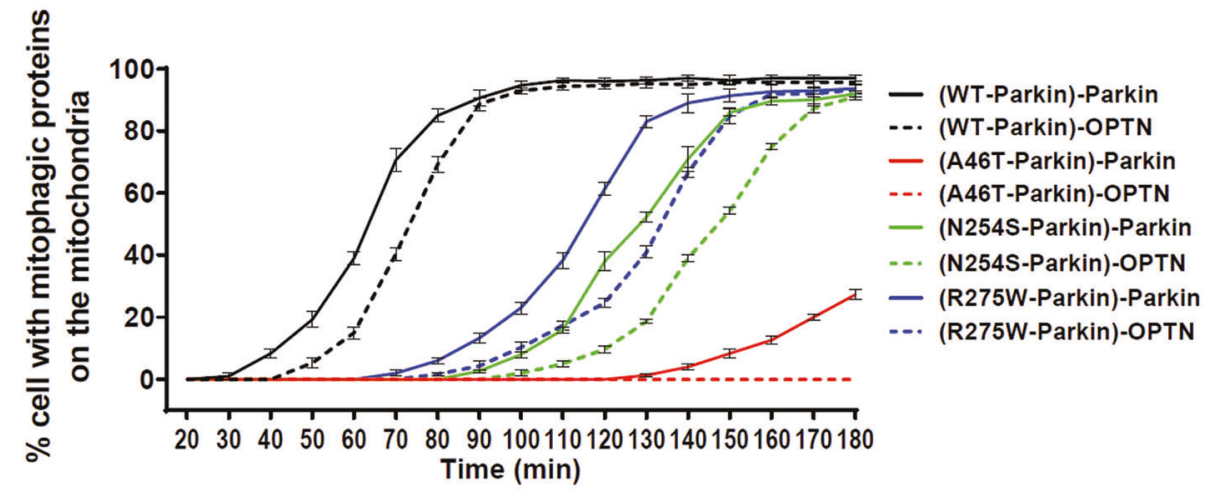

Fig. 3 The recruitment of the mitophagic receptor OPTN onto damaged mitochondria in mutant Parkin-expressing cells. a A549 cells were transfected with EGFP-OPTN and BFP-mito along with mCherry-tagged wild-type, N254S or A46T Parkin. Twenty-four hours later, cells were treated with $5 \mu \mathrm{M} \mathrm{A} / \mathrm{O}$ for 1,2 or $3 \mathrm{~h}$. Then, cells were fixed and subjected to an immunofluorescence assay. Scale bar: $15 \mu \mathrm{m}$. b Cells were transfected with EGFP-OPTN along with mCherry-tagged wild-type or mutant Parkin. Twenty-four hours later, cells were treated with $5 \mu \mathrm{M} \mathrm{A} / \mathrm{O}$. Cells with OPTN or Parkin on mitochondria were repeatedly counted at 10 min intervals. Data from three independent experiments are presented as the means \pm SDs 
a

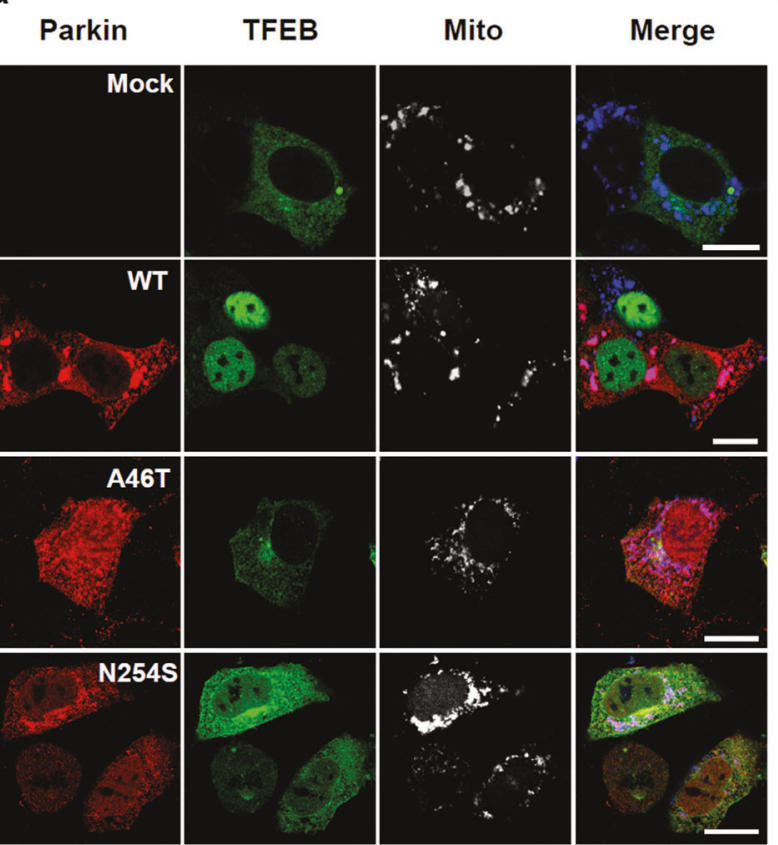

b Parkin Ub Mito Merge

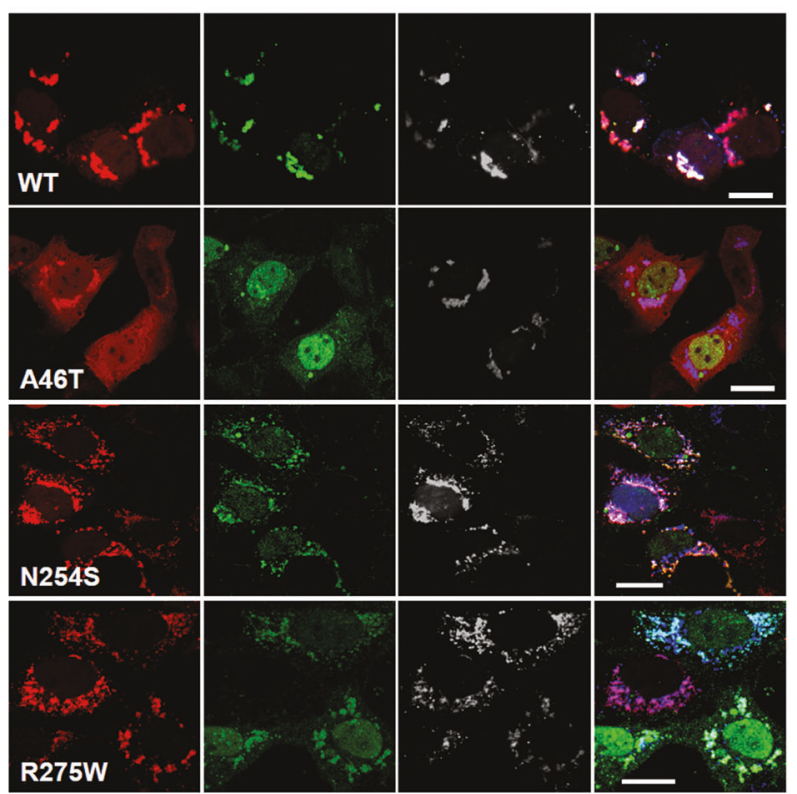

Fig. 4 Lung cancer-related mutant Parkin fails to regulate mitochondrial enrichment of ubiquitin (Ub) and nuclear translocation of TFEB. a A549 cells were transfected with TFEB-EGFP and BFP-mito in combination with mCherry-tagged wild-type, A46T or N254S Parkin. After 24 h, cells were treated with $5 \mu \mathrm{M} \mathrm{A} / O$ for $2 \mathrm{~h}$ and subjected to immunocytochemistry with an anti-FLAG antibody. Scale bars: $20 \mu \mathrm{m}$. b A549 cells were transfected with HA-Ub; BFP-mito; and mCherry-tagged wild-type, A46T, N254S or R275W Parkin for 24 h. Cells were treated with $5 \mu$ M A/ $\mathrm{O}$ for $4 \mathrm{~h}$. Then, cells were fixed and subjected to immunocytochemistry using anti-HA antibodies. Scale bars: $20 \mu \mathrm{m}$

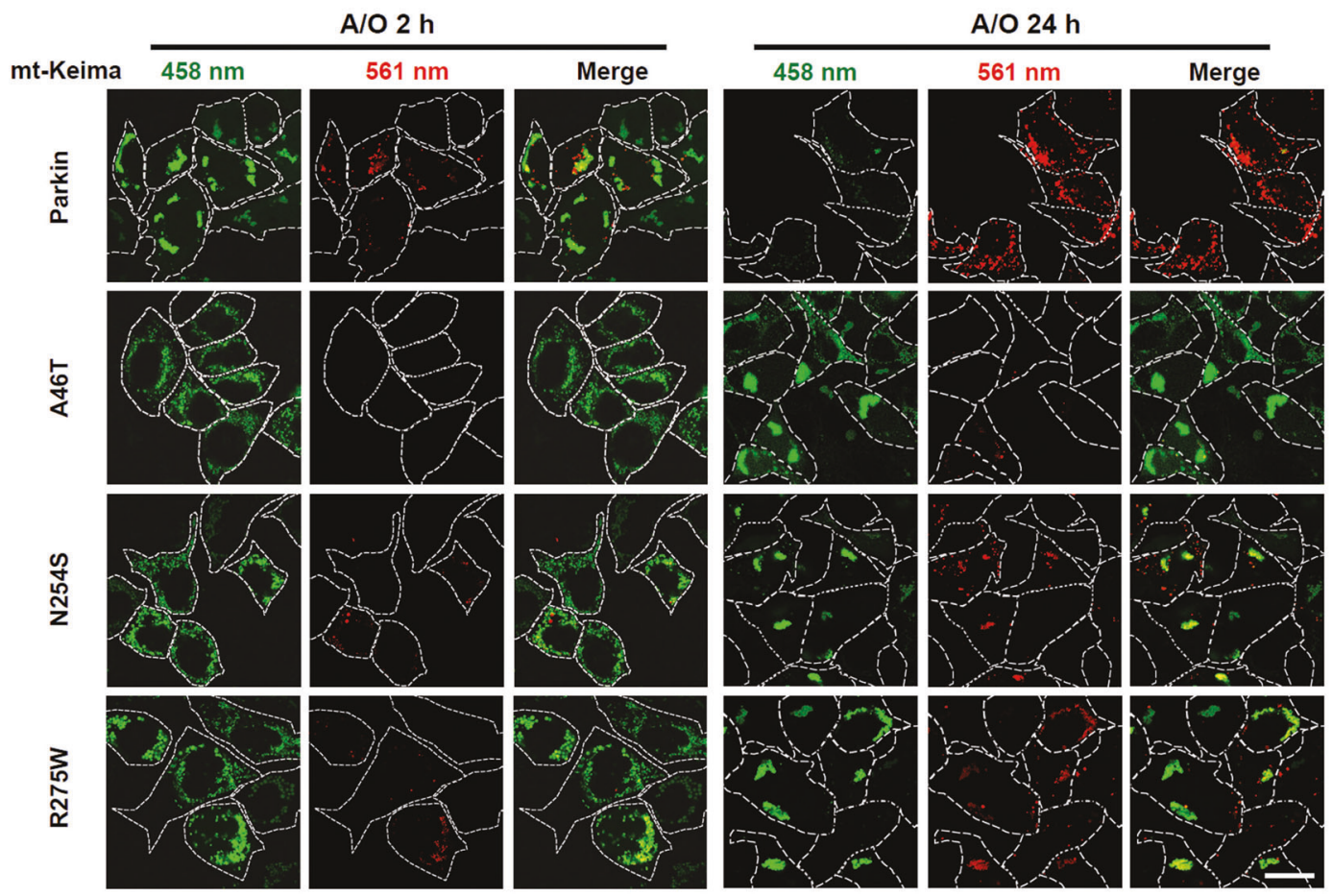

Fig. 5 Mutations in Parkin decrease mitophagic flux. A549 cells were transfected with mt-Keima along with FLAG-tagged wild-type or mutant Parkin. Twenty-four hours later, cells were treated with $5 \mu \mathrm{M} \mathrm{A} / \mathrm{O}$ for $2 \mathrm{~h}$ or $1 \mu \mathrm{M} \mathrm{A} / \mathrm{O}$ for $24 \mathrm{~h}$. Then, cells were observed using confocal microscopy. Scale bar: $15 \mu \mathrm{m}$

by increasing mitophagic signaling. Taken together, these data suggest that induction of mitophagy may be a potential treatment for lung cancer caused by Parkin loss-of-function mutations.

\section{DISCUSSION}

Autophagy plays a fundamental role in regulating the intracellular clearance of organelles, proteins and pathogens. Previous studies have shown that autophagy is tightly associated with a number of 
a

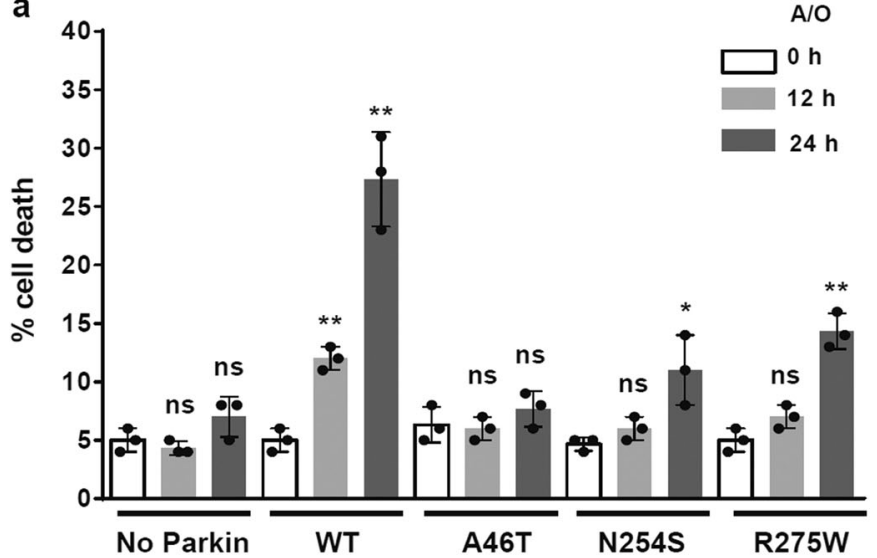

b

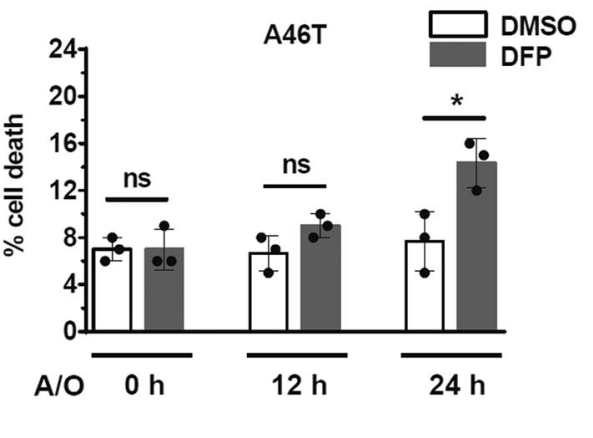

Fig. 6 Lung cancer-related mutations of Parkin affect cancer cell death caused by lethal mitophagy. a A549 cells were transfected with the indicated plasmids and treated with $1 \mu \mathrm{M} \mathrm{A} / \mathrm{O}$ for the indicated times. PI staining of Parkin-transfected cells with mitochondrial damage revealed cell death. The data are presented as the means \pm SDs. Comparisons were made relative to the $0 \mathrm{~h}$ condition in each group; $\mathrm{ns}$, no significance; ${ }^{*} P<0.05 ;{ }^{* \star} P<0.01$; $t$-test. b A549 cells were transfected with mCherry-A46T Parkin. Twenty-four hours later, cells were treated with $1 \mathrm{mM}$ deferiprone (DFP) for $24 \mathrm{~h}$ in combination with $1 \mu \mathrm{M} \mathrm{A} / \mathrm{O}$ for the indicated times. Then, cells were stained with PI to visualize cell death. Data from three experiments are presented as the means \pm SDs. ns, no significance; ${ }^{*} P<0.05 ;{ }^{* *} P<0.01 ; t$-test

human diseases, including neurodegenerative diseases, inflammatory diseases and cancer [14]. It is worth noting that studies of PINK1/Parkin-mediated mitophagy have mainly focused on neurodegenerative diseases, such as PD, whereas the pathogenic relationship between PINK1/Parkin-mediated mitophagy and cancer development remains elusive.

The current concept of mitophagy is that cells may use mitophagy to remove damaged mitochondria to protect themselves against toxicity generated by damaged mitochondria, such as reactive oxygen species (ROS). However, emerging evidence indicates that long-term induction of mitophagy in Parkin-expressing cells would lead to cell death through mechanisms currently unknown but possibly associated with apoptosis. For example, a previous report showed that ceramide, a known tumor suppressor widely used in preclinical and clinical studies in cancer research, could trigger cancer cell death and tumor suppression through lethal mitophagy [39]. Consistent with this finding, we found that DFP treatment significantly decreased the survival of lung cancer cell lines expressing pathogenic Parkin mutants (Fig. 6b). One issue in the field of autophagy is the lack of chemicals that can directly enhance downstream signaling in the pathway, such as inducers of autophagosome-lysosome fusion or autophagosome-substrate recognition. Given that DFP treatment increased the number of autolysosome-targeted mitochondria and benefits of the research progress made in the pharmacological regulation of mitophagy [40], our study not only is helpful for understanding the contribution of PINK1/Parkin-mediated mitophagy to the pathogenesis of lung cancer development but also offers experimental evidence for the effective treatment of lung cancer and possibly other types of human cancers.

Mechanistically, the A46T mutation may affect the conformation of the UBL domain of Parkin, thereby either inhibiting the phosphorylation of UBL or blocking UBL release, which, in turn, stops the subsequent activation of the Parkin E3 catalytic center. In addition, the N254S and R275W mutations are in the RING1 domain, which may inhibit the interaction between phosphorylated ubiquitin and the RING1 domain, thereby decreasing the E3 activity of Parkin. To date, it is not understood why both PD and cancer, two entirely different diseases, can be caused by inactivating mutations in Parkin. Additionally, it is intriguing that the single mutation R275W in PARK2 can either cause PD or cause cancer. There are several possibilities. First, non-cell-autonomous toxicity may also contribute to dopaminergic neuron degeneration in PD, since germline mutation in PARK2 will result in loss of Parkin function across all cell types, including glial cells such as microglia, astrocytes and oligodendrocytes, in the central nervous system. Second, various types of cellular stress in affected neurons or tumor cells may lead to different expression levels of mitophagy-related proteins. The combination of this variety and Parkin dysfunction may cause different biological consequences; therefore, the R275W germline mutation, which has already been shown to impair mitophagy [41], is associated with both PD and cancer. The last but perhaps the most important consideration is that dopaminergic neurons are more vulnerable when mitochondrial functions decline during aging. In support of this idea, Parkin-null mice did not develop a neurodegenerative phenotype under normal conditions, whereas they exhibited dopaminergic neuron-specific degeneration when exposed to mitochondrial stress [42]. Given that PD is a late-onset, age-related disease with increasing mitochondrial damage during disease progression, it is reasonable to postulate that Parkin-inactivating mutations would accelerate the accumulation of damaged mitochondria accompanied by high levels of toxic species, such as ROS, eventually resulting in the death of dopaminergic neurons and the development of PD during the aging process. Taken together, our findings suggest that both PD-linked and cancer-linked mutations in PARK2 can inhibit mitophagy in different ways, and strategies to enhance functional mitophagy may be novel treatments for both diseases.

\section{ACKNOWLEDGEMENTS}

This work was supported by the National Natural Science Foundation of China (Nos. 31771117, 31701222 and 31571053), the National Key Plan for Scientific Research and Development of China (No. 2017YFC0909100), a Project Funded by Jiangsu Key Laboratory of Neuropsychiatric Diseases (BM2013003) and a Project Funded by the Priority Academic Program Development of the Jiangsu Higher Education Institutes (PAPD).

\section{AUTHOR CONTRIBUTIONS}

ZY, HFW, MHS and QSL designed the experiments and drafted the manuscript; ZLZ, NNW, QLM, YC, LY and LZ performed the experiments.

\section{ADDITIONAL INFORMATION}

The online version of this article (https://doi.org/10.1038/s41401-019-0260-6) contains supplementary material, which is available to authorized users.

Competing interests: The authors declare no competing interests. 


\section{REFERENCES}

1. McWilliams TG, Muqit MM. PINK1 and Parkin: emerging themes in mitochondrial homeostasis. Curr Opin Cell Biol. 2017:45:83-91.

2. Harper JW, Ordureau A, Heo JM. Building and decoding ubiquitin chains for mitophagy. Nat Rev Mol Cell Biol. 2018;19:93-108.

3. Walden H, Muqit MM. Ubiquitin and Parkinson's disease through the looking glass of genetics. Biochem J. 2017;474:1439-51.

4. Zhang $C$, Lin $M$, Wu R, Wang $X$, Yang B, Levine AJ, et al. Parkin, a p53 target gene, mediates the role of $\mathrm{p} 53$ in glucose metabolism and the Warburg effect. Proc Natl Acad Sci USA. 2011;108:16259-64.

5. Fujiwara M, Marusawa H, Wang HQ, Iwai A, Ikeuchi K, Imai $Y$, et al. Parkin as a tumor suppressor gene for hepatocellular carcinoma. Oncogene. 2008;27: 6002-11.

6. Picchio MC, Martin ES, Cesari R, Calin GA, Yendamuri S, Kuroki T, et al. Alterations of the tumor suppressor gene Parkin in non-small cell lung cancer. Clin Cancer Res. 2004;10:2720-4.

7. Bernardini JP, Lazarou M, Dewson G. Parkin and mitophagy in cancer. Oncogene. 2017;36:1315-27.

8. Veeriah S, Taylor BS, Meng S, Fang F, Yilmaz E, Vivanco I, et al. Somatic mutations of the Parkinson's disease-associated gene PARK2 in glioblastoma and other human malignancies. Nat Genet. 2010;42:77-82.

9. Xiong D, Wang Y, Kupert E, Simpson C, Pinney SM, Gaba CR, et al. A recurrent mutation in PARK2 is associated with familial lung cancer. Am J Hum Genet. 2015;96:301-8

10. Yeo CW, Ng FS, Chai C, Tan JM, Koh GR, Chong YK, et al. Parkin pathway activation mitigates glioma cell proliferation and predicts patient survival. Cancer Res. 2012;72:2543-53.

11. Matsuda S, Nakanishi A, Minami A, Wada Y, Kitagishi Y. Functions and characteristics of PINK1 and Parkin in cancer. Front Biosci (Landmark Ed). 2015;20:491-501.

12. Vyas S, Zaganjor E, Haigis MC. Mitochondria and cancer. Cell. 2016;166:555-66.

13. Dikic I, Elazar Z. Mechanism and medical implications of mammalian autophagy. Nat Rev Mol Cell Biol. 2018;19:349-64.

14. Levine B, Kroemer G. Biological functions of autophagy genes: a disease perspective. Cell. 2019;176:11-42.

15. Wauer T, Simicek M, Schubert A, Komander D. Mechanism of phospho-ubiquitininduced PARKIN activation. Nature. 2015;524:370-4.

16. Kane LA, Lazarou M, Fogel Al, Li Y, Yamano K, Sarraf SA, et al. PINK1 phosphorylates ubiquitin to activate Parkin E3 ubiquitin ligase activity. J Cell Biol. 2014;205: 143-53.

17. Lazarou M, Sliter DA, Kane LA, Sarraf SA, Wang C, Burman JL, et al. The ubiquitin kinase PINK1 recruits autophagy receptors to induce mitophagy. Nature. 2015; 524:309-14.

18. Wong YC, Holzbaur EL. Optineurin is an autophagy receptor for damaged mitochondria in parkin-mediated mitophagy that is disrupted by an ALS-linked mutation. Proc Natl Acad Sci USA. 2014;111:E4439-48.

19. Moore AS, Holzbaur EL. Dynamic recruitment and activation of ALS-associated TBK1 with its target optineurin are required for efficient mitophagy. Proc Natl Acad Sci USA. 2016;113:E3349-58.

20. Nezich CL, Wang C, Fogel Al, Youle RJ. MiT/TFE transcription factors are activated during mitophagy downstream of Parkin and Atg5. J Cell Biol. 2015;210:435-50.

21. Allen GF, Toth R, James J, Ganley IG. Loss of iron triggers PINK1/Parkin-independent mitophagy. EMBO Rep. 2013;14:1127-35.

22. Ying Z, Wang H, Fan H, Zhu X, Zhou J, Fei E, et al. Gp78, an ER associated E3, promotes SOD1 and ataxin-3 degradation. Hum Mol Genet. 2009;18:4268-81.

23. Ying $Z$, Wang $H$, Fan $H$, Wang $G$. The endoplasmic reticulum (ER)-associated degradation system regulates aggregation and degradation of mutant neuroserpin. J Biol Chem. 2011;286:20835-44.
24. Wang $\mathrm{H}$, Ying Z, Wang G. Ataxin-3 regulates aggresome formation of copper-zinc superoxide dismutase (SOD1) by editing K63-linked polyubiquitin chains. J Biol Chem. 2012;287:28576-85.

25. Tao Z, Wang H, Xia Q, Li K, Jiang X, Xu G, et al. Nucleolar stress and impaired stress granule formation contribute to C9orf72 RAN translation-induced cytotoxicity. Hum Mol Genet. 2015;24:2426-41.

26. Xia Q, Wang G, Wang $\mathrm{H}, \mathrm{Hu}$ Q, Ying Z. Folliculin, a tumor suppressor associated with Birt-Hogg-Dube (BHD) syndrome, is a novel modifier of TDP-43 cytoplasmic translocation and aggregation. Hum Mol Genet. 2016;25:83-96.

27. Xia Q, Wang H, Hao Z, Fu C, Hu Q, Gao F, et al. TDP-43 loss of function increases TFEB activity and blocks autophagosome-lysosome fusion. EMBO J. 2016;35: 121-42.

28. Zhou L, Wang HF, Ren HG, Chen D, Gao F, Hu QS, et al. Bcl-2-dependent upregulation of autophagy by sequestosome $1 /$ p62 in vitro. Acta Pharmacol Sin. 2013;34:651-6.

29. Lv G, Sun D, Zhang J, Xie X, Wu X, Fang W, et al. Lx2-32c, a novel semi-synthetic taxane, exerts antitumor activity against prostate cancer cells in vitro and in vivo. Acta Pharm Sin B. 2017;7:52-8.

30. Liu D, Tang H, Li XY, Deng MF, Wei N, Wang X, et al. Targeting the HDAC2/HNF4A/miR-101b/AMPK pathway rescues tauopathy and dendritic abnormalities in Alzheimer's disease. Mol Ther. 2017;25:752-64.

31. Yang Y, Guan D, Lei L, Lu J, Liu JQ, Yang G, et al. H6, a novel hederagenin derivative, reverses multidrug resistance in vitro and in vivo. Toxicol Appl Pharm. 2018;341:98-105.

32. Wang X, Liu D, Huang HZ, Wang ZH, Hou TY, Yang X, et al. A novel microRNA-124/ PTPN1 signal pathway mediates synaptic and memory deficits in Alzheimer's disease. Biol Psychiatry. 2018;83:395-405.

33. Su Y, Deng MF, Xiong W, Xie AJ, Guo J, Liang ZH, et al. MicroRNA-26a/deathassociated protein kinase 1 signaling induces synucleinopathy and dopaminergic neuron degeneration in Parkinson's disease. Biol Psychiatry. 2019;85:769-81.

34. Wu JC, Qi L, Wang Y, Kegel KB, Yoder J, Difiglia M, et al. The regulation of $\mathrm{N}$-terminal Huntingtin (Htt552) accumulation by Beclin1. Acta Pharmacol Sin. 2012;33:743-51.

35. Ren ZX, Zhao YF, Cao T, Zhen XC. Dihydromyricetin protects neurons in an MPTPinduced model of Parkinson's disease by suppressing glycogen synthase kinase-3 beta activity. Acta Pharmacol Sin. 2016;37:1315-24.

36. Fang LM, Li B, Guan JJ, Xu HD, Shen GH, Gao QG, et al. Transcription factor EB is involved in autophagy-mediated chemoresistance to doxorubicin in human cancer cells. Acta Pharmacol Sin. 2017;38:1305-16.

37. Sun N, Malide D, Liu J, Rovira II, Combs CA, Finkel T. A fluorescence-based imaging method to measure in vitro and in vivo mitophagy using mt-Keima. Nat Protoc. 2017:12:1576-87.

38. Chen $Y, X u$ S, Wang N, Ma Q, Peng P, Yu Y, et al. Dynasore suppresses mTORC1 activity and induces autophagy to regulate the clearance of protein aggregates in neurodegenerative diseases. Neurotox Res. 2019; in press. https://doi.org/ 10.1007/s12640-019-00027-9.

39. Sentelle RD, Senkal CE, Jiang W, Ponnusamy S, Gencer S, Selvam SP, et al. Ceramide targets autophagosomes to mitochondria and induces lethal mitophagy. Nat Chem Biol. 2012;8:831-8.

40. Georgakopoulos ND, Wells G, Campanella M. The pharmacological regulation of cellular mitophagy. Nat Chem Biol. 2017;13:136-46.

41. Narendra D, Kane LA, Hauser DN, Fearnley IM, Youle RJ. p62/SQSTM1 is required for Parkin-induced mitochondrial clustering but not mitophagy; VDAC1 is dispensable for both. Autophagy. 2010;6:1090-106.

42. Pickrell AM, Huang $\mathrm{CH}$, Kennedy SR, Ordureau A, Sideris DP, Hoekstra JG, et al. Endogenous Parkin preserves dopaminergic substantia nigral neurons following mitochondrial DNA mutagenic stress. Neuron. 2015;87:371-81. 